that all patients currently taking dopamine receptor blocking agents (DRBAs) should be screened for TD, and that the Abnormal Involuntary Movement Scale (AIMS) is the standard structured assessment for monitoring severity of TD. There was consensus $(76 \%)$ that a semistructured assessment could be used for more frequent routine TD screening. Respondents unanimously agreed that treatment with first generation antipsychotics, older age, and longer cumulative exposure to antipsychotics were risk factors for TD. For TD diagnosis, consensus (89\%) was reached that a patient with an AIMS score $>2$ (mild) affecting 1 body area should be considered as having possible TD; consensus $(93 \%)$ was also reached that TD was most often evident in orofacial musculature, although other body areas may be affected and should not be neglected. Consensus was not reached on minimum cumulative duration of DRBA exposure for TD diagnosis, but a majority $(70 \%)$ agreed that minimum cumulative exposure of 1 month may be sufficient. For TD treatment, unanimity or consensus was reached on 4 strategies to consider: discussion of treatment options with patients/caregivers $(100 \%)$, modification of antipsychotic regimen $(100 \%)$, treatment with VMAT2 inhibitor $(100 \%)$, and modification of anticholinergic regimen $(86 \%)$.

CONCLUSIONS: Using a Nominal Group and modified Delphi process, consensus was reached within 1-2 rounds on several key aspects of TD screening, diagnosis, and treatment. This process may offer an expedient method to identify gaps in agreement and facilitate updated management guidelines.

Funding Acknowledgements: Sponsored by Neurocrine Biosciences, Inc.

42

\section{Training Forgiveness - A Novel Approach to Reducing Physician Burnout}

Lidia Firulescu, M.D. '; Ross W. May, Ph.D. ${ }^{2}$; Frank D. Fincham, Ph.D. ${ }^{3}$; Emelina A. Arocha, M.D.; FAPA ${ }^{4}$, and Marcos A. Sanchez-Gonzalez, M.D., Ph.D. ${ }^{5}$

${ }^{1}$ Department of Psychiatry, Larkin Community

Hospital, South Miami, FL

${ }^{2}$ Family Institute, Florida State University, Tallahassee, FL

${ }^{3}$ Family Institute, Florida State University, Tallahassee, FL

${ }^{4}$ Department of Psychiatry, Larkin Community

Hospital, South Miami, FL

${ }^{5}$ Division of Clinical \& Translational Research, Larkin Community Hospital, South Miami, FL
ABSTRACT: Study Objective: Psychological risk factors that lead to impaired work performance, negatively impacting mental and physical health, have emerged as a concern across clinical settings. Although depression and anxiety are linked to poor physician mental health, physician burnout characterized by work related stress due to chronic exhaustion from clinical work, cynicism toward meaning of the medical profession, and feelings of inadequacy toward work related accomplishments, may be an even stronger indicator of well-being. Literature suggests that work satisfaction among physicians is rapidly deteriorating owing to high rates of burn out and poor mental health. Although the relationship between work burnout (WB) and negative affectivity has been well documented, the association with positive affect, such as trait forgiveness (TF) has been overlooked. On that note, research shows that lifetime stress severity and lower levels of forgiveness predict worse mental and physical health. Since TF has been linked strongly with healthy workplace relationships, positive occupational outcomes and general well-being, its association with WB remains to be investigated. Therefore, the aim of the present study was to explore the link between TF and WB among physicians. We hypothesized that TF would be associated with reduced levels of burnout.

METHOD: A total of $62(\mathrm{~F}=23)$ medical residents at a Teaching Hospital consented for the study. Residents were administered surveys on WB (Maslach BurnoutInventory), workplace bullying, personal bullying (PB), interpersonal rejection sensitivity (IRS), perceived stress scale (PSS), TF, anxiety, and depression, all of which were anonymously submitted via electronically. Hierarchical multiple regression (HMR) models were used to determine the associations between WB, work environment social factors and TF. A p-value of $<0.05$ was considered significant.

RESULTS: The mean age $33.1 \pm \mathrm{SD} 4.2$ years. HMR analysis using WB as main outcome contained 6 predictors: Model 1 contained depression and anxiety, Model 2 added PB, Model 3 added IRS and PSS, Model 4 added TF. Anxiety and TF were the only significant predictors $(\mathrm{p}=>0.05)$ accounting for $10.4 \%$ and $17.5 \%$ of the variance in WB scores, respectively.

CONCLUSIONS: The novel finding of the present study is that TF was associated with low levels of burnout. Additionally, WB was found to be linked to anxiety and depression which is in line with previous research. These data suggest that $\mathrm{TF}$ could be a potential resolution to the deleterious influence of burnout. Further exploration is needed in order to understand the psychology of forgiveness as a potential adjuvant and/or therapeutic 
intervention for physicians' burnout. These results suggest that strategies including forgiveness training aimed at decreasing WB while increasing job satisfaction among physicians warrant further exploration. Funding Acknowledgements: no funding

43

\section{Tinnitus as an Aura for Sleep Paralysis}

Luvleen Shergill, MS4'; Jasir Nayati, MS4'ㄹ Reshma Nair, $\mathrm{MS4}^{3}$; and Alan R. Hirsch, $M D^{4}$

${ }^{1}$ Aureus University School of Medicine, Oranjestad, Aruba

${ }^{2}$ St. James School of Medicine, Anguilla

${ }^{3}$ American University of Integrative Sciences, Barbados

${ }^{4}$ Smell and Taste Treatment and Research Foundation, Chicago, IL

ABSTRACT: Objective: To understand that tinnitus may be an aura for sleep paralysis.

BACKGROUND: Sleep paralysis is a transient-paralysis which occurs during awakening or falling asleep (Wilson, 1928). Those affected experience symptoms including visual, auditory, and haptic hallucinations, voluntary motor paralysis with intact ocular and respiratory motor movements, and diffuse or localized paresthesias. Sleep paralysis associated with tinnitus as an aura, has not heretofore been described.

METHODS: A 34 year-old, right-handed female presented with a 13 year history of sleep paralysis. One month prior, she began to notice tinnitus prior to the onset of sleep paralysis. The tinnitus was bilateral, high-pitched, with a volume intensity of $5 / 10$, lasting seven seconds prior to sleep initiation. She denied hearing loss, vertigo, dizziness, cataplexy, deja vu and jamais vu. After termination of tinnitus, she experienced paresthesia, "like at a dentist's office" radiating from her posterior neck, to her tongue and down to her toes. She described seeing a white-shadowy male figure moving around her room, lasting seven seconds. Accompanied by a masculine "ahh" sound, lasting for three seconds. The sleep paralysis occurred after these events, lasting up to eight hours, or until her husband wakes her.

RESULTS: Abnormalities in Physical Examination: General Examination: right arm hemangioma 4 by $5 \mathrm{~cm}$. Reflexes: absent bilateral brachioradialis, $1+$ bilateral quadriceps femoris and bilateral Achilles tendon. Neuropsychiatric Examination: Calibrated Finger Rub Auditory Screening Test: faint $70 \mathrm{AU}$ (normal).

DISCUSSION: Tinnitus has been described as an aura for migraines (Schankin, 2014), temporal lobe epilepsy (TLE)
(Florindo, 2006), and narcolepsy-cataplexy (Marco, 1978). These epochs may represent amigranous migraines, which initially present with tinnitus that occurs both during the day and night, forcing the patient to be partially awoken at night with induction of the sleep paralysis sequence. It would be worthwhile to query those with narcolepsy or sleep paralysis if tinnitus precedes the event.

44

Real World Effectiveness: A 6-month Naturalistic Follow-up Study of Schizophrenia Patients After Switching to Aripiprazole Monohydrate (AOM) Treatment

Marcos Gómez-Revuelta, MD-PhD ${ }^{1,2}$; José María Pelayo-Terán, MD-PhD ${ }^{1,2,3}$; María Juncal-Ruiz, $M D^{1,2}$, María Fernández-Rodríguez, $M D^{1,2}$; Javier VázquezBourgon, MD-PhD ${ }^{1,2}$; Paula Suárez-Pinilla, $\mathrm{MD}^{1,2}$; and Oriol Porta-Olivares, $M D^{1,2}$

${ }^{1}$ University Hospital Marqués de Valdecilla, IDIVAL, Department of Psychiatry, School of Medicine, University of Cantabria, Santander, Spain

${ }^{2}$ CIBERSAM, Centro Investigación Biomédica en Red Salud Mental, Madrid, Spain

${ }^{3}$ Unidad de Gestión Clínica de Psiquiatría y Salud Mental. Hospital El Bierzo. Servicio de Salud de Castilla y León (SACYL), Ponferrada (León), Spain

ABSTRACT: Rationale: Long-acting injectable antipsychotic therapies may offer benefits over oral antipsychotics in patients with schizophrenia. However, there is still a lack of real-world studies assessing the effectiveness of these therapies.

OBJECTIVE: This study aimed to explore the safety, tolerability, and treatment response of aripiprazole monohydrate (AOM) once monthly in non-acute but symptomatic adult patients switched from previous therapy with frequently used oral or injectable atypical antipsychotics.

METHODS: This was a post hoc analysis of a prospective, interventional, single-arm, open-label, 6-month study.

RESULTS: The patients $(\mathrm{N}=54)$ were switched to aripiprazole monohydrate once-monthly (AOM) from daily oral treatment or monthly injectable treatment with either aripiprazole $(n=25)$, olanzapine $(n=7)$, paliperidone extended-release (PP1M) $(n=10)$, quetiapine $(\mathrm{n}=4)$, or risperidone $(\mathrm{n}=8)$. In all groups, mean Positive and Negative Syndrome Scale total $(\mathrm{p}=0.0001)$ and Clinical Global Impression-Severity scores improved significantly $(\mathrm{p}=0.0001)$. A reduction of $\geq 50 \%$ reduction of BPRS total-score and a CGI 